\title{
Turkish folk medicinal plants, VIII: Lalapaşa (Edirne)
}

\author{
Ertan Tuzlacı, Duygu Fatma Alparslan İşbilen, Gizem Bulut
}

ABSTRACT: In this study, the folk medicinal plants of Lalapaşa (Edirne) were researched. During the field works, the information were obtained from local healers, experienced adults and patients by personal interviews and the specimens of the plants were collected. According to the results of the identifications of the specimens, 55 plant taxa are used in therapy in Lalapaşa. These are presented in a table in the text. Among them 44 taxa are wild and 11 taxa are cultivated plants. The folk medicinal plants are mostly used for stomach ailments, hemorrhoids, diabetes, cold and warts.

KEY WORDS: Folk medicine, Medicinal plants, Lalapaşa, Edirne, Turkey

\section{INTRODUCTION}

Lalapaşa (Edirne) is located near the TurkishBulgarian border in the European part of Turkey (Figure-1). It covers an area of $536 \mathrm{~km}^{2}$ and its total population is $10.154(1)$.

Agricultural of various plants (wheat, sunflower and barley) and animal breeding have an important role in the popular life in Lalapaşa.

This is the first comprehensive ethnobotanical research realized between 2004-2006. However, this study includes only the plants used in traditional folk medicine.

\section{MATERIAL AND METHODS}

The information about the various data (local names, part(s) of plants used, ailments treated, therapeutic effect, methods of preparation, methods of administration and duration of the treatment) were obtained from local healers, experienced adults and patients by personal interviews.

The plant specimens are kept in the Herbarium of the Faculty of Pharmacy, Marmara University (MARE). The "Flora of Turkey and the East
Aegean Islands" (2) was mainly used for the identification of the plants.

\section{RESULTS AND DISCUSSION}

During the study, 151 specimens were collected in the area. According to the results of identifications, 55 taxa are being used as folk medicine in Lalapaşa. Among them 44 taxa are wild and 11 taxa are cultivated plants. These are presented in Table-1 arranged in alphabetical order of their botanical names with the relevant information.

The folk medicinal plants are mostly used for stomach ailments, hemorrhoids, diabetes, cold and warts.

Decoction and infusion are the methods mostly used for the preparation of the folk medicine.

The comparison of the folk medicinal plants recorded in the ethnobotanical studies which were made in the European part of Turkey (3-6) is presented in Table 2. This comparison comprises only the plants which were recorded in two or more studies. According to this table, the usages of only two plants (Ecballium elaterium and Prunus spinosa subsp. dasyphylla) are similar in five studies.
AFFILIATIONS

Marmara Üniversitesi

Eczacılık Fakültesi

Farmasötik Botanik Anabilim

Dalı, İstanbul, Türkiye

CORRESPONDENCE

Ertan Tuzlacl

E-mail:

ertan.tuzlaci@marmara.

edu.tr

Received:

December 10, 2009

Revision:

December 18, 2009

Accepted:

December 18, 2009 
TABLE 1. Folk medicinal plants of Lalapaşa (Edirne, Turkey)

\begin{tabular}{|c|c|c|c|c|c|}
\hline Botanical name and Voucher specimen & Local names & Parts used & Uses/Ailments treated & Preparations & Modes of use \\
\hline $\begin{array}{l}\text { Achillea crithmifolia Waldst. et Kit. } \\
\text { (Asteraceae), MARE } 9404,10249\end{array}$ & $\begin{array}{l}\text { Civanperçemi, } \\
\text { Mayasıl otu }\end{array}$ & $\begin{array}{l}\text { Aerial parts } \\
\text { Aerial parts } \\
\text { Aerial parts } \\
\text { Aerial parts }\end{array}$ & $\begin{array}{l}\text { Hemorrhoids } \\
\text { Boil (on hand) } \\
\text { Heart diseases } \\
\text { Nervous system diseases }\end{array}$ & $\begin{array}{l}\text { Infusion } \\
\text { Infusion } \\
\text { Infusion } \\
\text { Infusion }\end{array}$ & $\begin{array}{l}\text { Int. } \\
\text { Int. } \\
\text { Int. } \\
\text { Int. }\end{array}$ \\
\hline $\begin{array}{l}\text { (Liliaceae) } \\
\text { Allium sativum L. }\end{array}$ & Sarımsak & Bulbils & Hair restorer & Crushed & Ext. \\
\hline $\begin{array}{l}\text { Carduus nutans L. } \\
\text { (Asteraceae), MARE } 10302\end{array}$ & $\begin{array}{l}\text { Eşek çalısı, } \\
\text { Eşek dikeni }\end{array}$ & $\begin{array}{l}\text { Flowering } \\
\text { branches }\end{array}$ & Prostate ailments & Decoction & Int. \\
\hline $\begin{array}{l}\text { Centaurea solstitialis L. subsp. solstitialis } \\
\text { (Asteraceae), MARE } 9463,10274\end{array}$ & $\begin{array}{l}\text { Çakır dikeni, } \\
\text { Kavgalaz dikeni, } \\
\text { Kaynanadili }\end{array}$ & $\begin{array}{l}\text { Capitula } \\
\text { Capitula } \\
\text { Capitula }\end{array}$ & $\begin{array}{l}\text { Malaria } \\
\text { Malaria } \\
\text { Malaria }\end{array}$ & $\begin{array}{l}\text { Crushed } \\
\text { Crushed }\end{array}$ & $\begin{array}{l}\text { Int. } \\
\text { Int. } \\
\text { Int. }\end{array}$ \\
\hline $\begin{array}{l}\text { Cerasus mahaleb (L.) Miller } \\
\text { var. mahaleb } \\
\text { (Rosaceae), MARE 9408, } 9453\end{array}$ & $\begin{array}{l}\text { Delice kiraz, } \\
\text { Yaban kirazı }\end{array}$ & Seeds & Abdominal pain & Crushed & Int. \\
\hline $\begin{array}{l}\text { Cichorium intybus L. } \\
\text { (Asteraceae), MARE } 9456,10280\end{array}$ & Karakavuk & Latex & Wart & - & Ext. \\
\hline $\begin{array}{l}\text { Corylus avellana L. var. avellana } \\
\text { (Corylaceae), MARE } 9472\end{array}$ & Findık & Leaves & Prostate ailments & Decoction & Int. \\
\hline $\begin{array}{l}\text { Cornus mas L. } \\
\text { (Cornaceae), MARE } 9425\end{array}$ & Kızılcık & $\begin{array}{l}\text { Sap provided } \\
\text { from heated } \\
\text { branches }\end{array}$ & Wart & - & $\begin{array}{l}\text { Ext. (after } \\
\text { Crataegus monogyna } \\
\text { subsp. azarella } \\
\text { application) }\end{array}$ \\
\hline $\begin{array}{l}\text { Cotinus coggyria Scop. } \\
\text { (Anacardiaceae), MARE } 9418\end{array}$ & Tetre & $\begin{array}{l}\text { Leaves } \\
\text { Leaves } \\
\text { Leaves } \\
\text { Leaves } \\
\text { Leaves } \\
\text { Leaves } \\
\text { Leaves }\end{array}$ & $\begin{array}{l}\text { Foot and mouth disease (FMD) } \\
\text { Eczema (human\&animal) } \\
\text { Eczema } \\
\text { Rheumatism } \\
\text { Prostate ailments } \\
\text { Hemorrhoids } \\
\text { Calcification }\end{array}$ & $\begin{array}{l}\text { Infusion } \\
\text { Infusion } \\
\text { Decoction } \\
\text { Infusion } \\
\text { Decoction } \\
\text { Decoction } \\
\text { Decoction }\end{array}$ & $\begin{array}{l}\text { Ext. } \\
\text { Ext. } \\
\text { Ext. } \\
\text { Int. } \\
\text { Int., } 2 \times 1 \\
\text { Int. } \\
\text { Ext. }\end{array}$ \\
\hline $\begin{array}{l}\text { Crataegus monogyna Jacq. subsp. } \\
\text { azarella (Gris.) Franco } \\
\text { (Rosaceae), MARE } 9395\end{array}$ & Yemişgen & Spine & Wart & - & $\begin{array}{l}\text { Prickled (then used } \\
\text { Cornus mas) }\end{array}$ \\
\hline $\begin{array}{l}\text { Crataegus monogyna Jacq. } \\
\text { subsp. monogyna } \\
\text { (Rosaceae), MARE } 9451\end{array}$ & Yemişgen çalısı & Spine & Snake bite (for animals) & - & Prickled into bitten area \\
\hline $\begin{array}{l}\text { ACydonia oblonga Miller } \\
\text { (Rosaceae), MARE } 9440\end{array}$ & Ayva & $\begin{array}{l}\text { Leaves } \\
\text { Leaves } \\
\text { Leaves } \\
\text { Leaves }\end{array}$ & $\begin{array}{l}\text { Abdominal pain } \\
\text { Cold } \\
\text { Antitussive } \\
\text { Diabetes }\end{array}$ & $\begin{array}{l}\text { Decoction } \\
\text { Decoction } \\
\text { Infusion } \\
\text { Infusion }\end{array}$ & $\begin{array}{l}\text { Int. } \\
\text { Int. } \\
\text { Int. } \\
\text { Int. }\end{array}$ \\
\hline $\begin{array}{l}\text { Ecballium elaterium (L.) A. Rich. } \\
\text { (Cucurbitaceae), MARE } 9411\end{array}$ & $\begin{array}{l}\text { Acı kavun, } \\
\text { Mayasıl otu }\end{array}$ & $\begin{array}{l}\text { Fruit juice } \\
\text { Fruit juice } \\
\text { Fruit juice }\end{array}$ & $\begin{array}{l}\text { Sinusitis } \\
\text { Rheumatism } \\
\text { Hemorrhoids }\end{array}$ & $\begin{array}{l}\text { Boiled } \\
\text { Boiled } \\
-\end{array}$ & $\begin{array}{l}\text { Inhaled } \\
\text { Inhaled } \\
\text { Int. }\end{array}$ \\
\hline $\begin{array}{l}\text { Euphorbia nicaeensis All. subsp. } \\
\text { glareosa (Pallas ex Bieb.) A.R. Smith } \\
\text { var. lasiocarpa Boiss. } \\
\text { (Euphorbiaceae), MARE } 10287\end{array}$ & Sütlü ot & Whole plant & $\begin{array}{l}\text { Increase milk secrection (for } \\
\text { animal) }\end{array}$ & Int. & Int. \\
\hline $\begin{array}{l}\text { A Ficus carica L. subsp. carica } \\
\text { (Moraceae), MARE } 9419\end{array}$ & İncir & $\begin{array}{l}\text { Leaves } \\
\text { Leaves } \\
\text { Latex }\end{array}$ & $\begin{array}{l}\text { Abdominal pain } \\
\text { Hemorrhoids } \\
\text { Wart }\end{array}$ & $\begin{array}{l}\text { Decoction } \\
\text { Infusion } \\
-\end{array}$ & $\begin{array}{l}\text { Int. } \\
\text { Int. } \\
\text { Ext. }\end{array}$ \\
\hline $\begin{array}{l}\text { Fraxinus ornus L. subsp. ornus } \\
\text { (Oleaceae), MARE } 9434\end{array}$ & Dışbudak & $\begin{array}{l}\text { Stem bark } \\
\text { Shoots }\end{array}$ & $\begin{array}{l}\text { Hemorrhoids } \\
\text { Chicken diseases }\end{array}$ & $\begin{array}{l}\text { Decoction } \\
\text { (+sugar) } \\
\text { Decoction }\end{array}$ & $\begin{array}{l}\text { Int., before breakfast } \\
\text { Int. }\end{array}$ \\
\hline $\begin{array}{l}\text { Hyoscyamus niger L. } \\
\text { (Solanaceae), MARE 9393, } 10246\end{array}$ & Tatala & Fruits & Gum diseases & Boiled & Inhaled \\
\hline $\begin{array}{l}\text { Hypericum perforatum L. } \\
\text { (Hypericaceae), MARE } 9446,9462\end{array}$ & Sarı kantoron & $\begin{array}{l}\text { Aerial parts } \\
\text { Aerial parts } \\
\text { Flowering } \\
\text { branches }\end{array}$ & $\begin{array}{l}\text { Diabetes } \\
\text { Wound } \\
\text { Kidney ailments }\end{array}$ & $\begin{array}{l}\text { Decoction } \\
\text { Oleat } \\
\text { Infusion }\end{array}$ & $\begin{array}{l}\text { Int. } \\
\text { Ext. } \\
\text { Int. }\end{array}$ \\
\hline $\begin{array}{l}\text { A Juglans regia L. } \\
\text { (Juglandaceae), MARE } 9399\end{array}$ & Ceviz & $\begin{array}{l}\text { Leaves } \\
\text { Fruits } \\
\text { (immature) }\end{array}$ & $\begin{array}{l}\text { Headache } \\
\text { Hemorrhoids }\end{array}$ & $\begin{array}{l}\text { Crushed, } \\
\text { wrapped in a } \\
\text { cloth } \\
\text { Decoction }\end{array}$ & $\begin{array}{l}\text { Ext. } \\
\text { Int. }\end{array}$ \\
\hline $\begin{array}{l}\text { Lavatera thuringiaca } \mathrm{L} . \\
\text { (Malvaceae), MARE } 10283\end{array}$ & Fatmecik çiçeği & Flowers & Antitussive & Infusion & Int. \\
\hline
\end{tabular}

$\boldsymbol{\Delta}$ Cultivated plant; + mixed with; Int. = internal use; Ext. = external use; $2 \times 1=$ twice a day; it means 1 glass of decoction or infusion indicated. 
TABLE 1. Folk medicinal plants of Lalapaşa (Edirne, Turkey) (continued)

\begin{tabular}{|c|c|c|c|c|c|}
\hline Botanical name and Voucher specimen & Local names & Parts used & Uses/Ailments treated & Preparations & Modes of use \\
\hline $\begin{array}{l}\text { Malva sylvestris L. } \\
\text { (Malvaceae), MARE } 9413\end{array}$ & Ebe gümeci & $\begin{array}{l}\text { Aerial parts } \\
\text { Aerial parts } \\
\text { Flowers }\end{array}$ & $\begin{array}{l}\text { Hemorrhoids } \\
\text { Immunostimulan } \\
\text { Antitussive }\end{array}$ & $\begin{array}{l}\text { Decoction } \\
\text { Infusion } \\
\text { Infusion }\end{array}$ & $\begin{array}{l}\text { Int. } \\
\text { Int. } \\
\text { Int. }\end{array}$ \\
\hline $\begin{array}{l}\text { Matricaria chamomilla } \mathrm{L} \text {. } \\
\text { var. recutita }(\mathrm{L} \text {.) Grierson } \\
\text { (Asteraceae), MARE } 9433\end{array}$ & Papatya & $\begin{array}{l}\text { Capitulum } \\
\text { Capitulum }\end{array}$ & $\begin{array}{l}\text { Hemorrhoids } \\
\text { Diabetes }\end{array}$ & $\begin{array}{l}\text { Infusion } \\
\text { Infusion }\end{array}$ & $\begin{array}{l}\text { Int. } \\
\text { Int. }\end{array}$ \\
\hline $\begin{array}{l}\text { Melissa officinalis L. } \\
\text { (Lamiaceae), MARE } 9396\end{array}$ & Oğul otu & Aerial parts & Embolism & Infusion & Int., before breakfast \\
\hline $\begin{array}{l}\text { Mentha spicata L. subsp. spicata } \\
\text { (Lamiaceae), MARE } 9431\end{array}$ & Nane & $\begin{array}{l}\text { Aerial parts } \\
\text { Aerial parts }\end{array}$ & $\begin{array}{l}\text { Nausea } \\
\text { Stomachache }\end{array}$ & Infusion & $\begin{array}{l}\text { Int. } \\
\text { Int. }\end{array}$ \\
\hline $\begin{array}{l}\text { AMomordica charantia L. } \\
\text { (Cucurbitaceae) }\end{array}$ & $\begin{array}{l}\text { Yağanda, } \\
\text { Yarhanda }\end{array}$ & $\begin{array}{l}\text { Fruits } \\
\text { Fruits }\end{array}$ & $\begin{array}{l}\text { Wound } \\
\text { Stomachache }\end{array}$ & $\begin{array}{l}\text { Oleat } \\
\text { Oleat }\end{array}$ & $\begin{array}{l}\text { Ext. } \\
\text { Int., before breakfast }\end{array}$ \\
\hline \multirow[t]{2}{*}{$\begin{array}{l}\text { AMorus alba L. } \\
\text { (Moraceae), MARE } 9397\end{array}$} & Dut & Leaves & Panaris & $\begin{array}{l}\text { Wrapped in a } \\
\text { cloth }\end{array}$ & Ext. \\
\hline & & Leaves & Antitussive & Infusion & Int. \\
\hline $\begin{array}{l}\text { A Ocimum basilicum L. } \\
\text { (Lamiaceae) }\end{array}$ & Fesleğen & Aerial parts & Stomachache & Infusion & Int. \\
\hline $\begin{array}{l}\text { Onopordum tauricum Willd. } \\
\text { (Asteraceae), MARE 9394, } 10266\end{array}$ & $\begin{array}{l}\text { Eşek çalısı, } \\
\text { Eşek dikeni, } \\
\text { Deve dikeni }\end{array}$ & $\begin{array}{l}\text { Capitula } \\
\text { Aerial parts }\end{array}$ & $\begin{array}{l}\text { Burn } \\
\text { Liver ailments }\end{array}$ & $\begin{array}{l}\text { Oleat } \\
\text { Decoction }\end{array}$ & $\begin{array}{l}\text { Ext. } \\
\text { Int. }\end{array}$ \\
\hline $\begin{array}{l}\text { Origanum vulgare L. } \\
\text { subsp. vulgare } \\
\text { (Lamiaceae), MARE 9442, } 9474\end{array}$ & $\begin{array}{l}\text { Çay otu, } \\
\text { Kantız, } \\
\text { Kır çayı }\end{array}$ & $\begin{array}{l}\text { Aerial parts } \\
\text { Aerial parts } \\
\text { Aerial parts }\end{array}$ & $\begin{array}{l}\text { Stomach ailments } \\
\text { Cold } \\
\text { Strenghthen of bones }\end{array}$ & $\begin{array}{l}\text { Infusion } \\
\text { Infusion } \\
\text { Infusion }\end{array}$ & $\begin{array}{l}\text { Int. } \\
\text { Int. } \\
\text { Int. }\end{array}$ \\
\hline $\begin{array}{l}\text { Paliurus spina-christi Miller } \\
\text { (Zygophyllaceae), MARE } 9447\end{array}$ & Kara çalı & $\begin{array}{l}\text { Fruits } \\
\text { Fruits } \\
\text { Fruits } \\
\text { Fruits }\end{array}$ & $\begin{array}{l}\text { Heart diseases } \\
\text { Diabetes } \\
\text { Kidney stones } \\
\text { Abdominal pain }\end{array}$ & $\begin{array}{l}\text { Decoction } \\
\text { Decoction } \\
\text { Decoction } \\
\text { Decoction }\end{array}$ & $\begin{array}{l}\text { Int. } \\
\text { Int. } \\
\text { Int. } \\
\text { Int. }\end{array}$ \\
\hline $\begin{array}{l}\text { Papaver rhoeas L. } \\
\text { (Papaveraceae), MARE } 10267\end{array}$ & Gelincik & Petals & Imsomnia & $\begin{array}{l}\text { Put in water for } \\
1 \text { week }\end{array}$ & Int. \\
\hline$\Delta$ Persica vulgaris Miller (Rosaceae) & Şeftali & Leaves & Anthelmentic & Decoction & Int. \\
\hline $\begin{array}{l}\text { Pinus sylvestris } L \text {. } \\
\text { (Pinaceae), MARE } 9458\end{array}$ & Çam & Buds & Asthma & Decoction & Int. \\
\hline $\begin{array}{l}\text { Plantago lanceolata L. } \\
\text { (Plantaginaceae), MARE } 10269\end{array}$ & Sinirli ot & Aerial parts & Diarrhea (for children) & Decoction & Int. \\
\hline $\begin{array}{l}\text { Plantago major L. subsp. major } \\
\text { (Plantaginaceae), MARE } 9414,9436,9470\end{array}$ & $\begin{array}{l}\text { Sinirli ot, } \\
\text { Sinirli yaprak }\end{array}$ & $\begin{array}{l}\text { Aerial parts } \\
\text { Leaves }\end{array}$ & $\begin{array}{l}\text { Stomachache } \\
\text { Diabetes }\end{array}$ & $\begin{array}{l}\text { Decoction } \\
\text { Decoction }\end{array}$ & $\begin{array}{l}\text { Int. } \\
\text { Int. }\end{array}$ \\
\hline $\begin{array}{l}\text { Prunus spinosa L. subsp. dasyphylla } \\
\text { (Schur) Domin } \\
\text { (Rosaceae), MARE } 10236\end{array}$ & Güvem eriği & Fruits & Diabetes & Decoction & Int. \\
\hline $\begin{array}{l}\text { A Ricinus communis L. } \\
\text { (Euphorbiaceae), MARE } 9443\end{array}$ & $\begin{array}{l}\text { Bostankenesi, } \\
\text { Domuzkenesi }\end{array}$ & Seeds & Purgative (for animals) & $\begin{array}{l}\text { Added into the } \\
\text { feed }\end{array}$ & Int. \\
\hline $\begin{array}{l}\text { ARobinia pseudoacacia L. } \\
\text { (Fabaceae), MARE } 9393\end{array}$ & Katırtırnağı & Flowers & Bronchitis & Infusion & Int. \\
\hline $\begin{array}{l}\text { Rosa canina L. } \\
\text { (Rosaceae), MARE } 9468\end{array}$ & Kuşburnu & $\begin{array}{l}\text { Flowering } \\
\text { branches } \\
\text { Flowering } \\
\text { branches } \\
\text { Fruits } \\
\text { Fruits } \\
\text { Fruits }\end{array}$ & $\begin{array}{l}\text { Embolism } \\
\text { Heart diseases } \\
\text { Asthma } \\
\text { Antitussive } \\
\text { Cold }\end{array}$ & $\begin{array}{l}\text { Infusion } \\
\text { Infusion } \\
\text { Decoction } \\
\text { Decoction } \\
\text { Decoction }\end{array}$ & $\begin{array}{l}\text { Int. } \\
\text { Int. } \\
\text { Int. } \\
\text { Int. } \\
\text { Int. }\end{array}$ \\
\hline $\begin{array}{l}\text { Rubia tictorum L. } \\
\text { (Rubiaceae), MARE } 10252\end{array}$ & Yapışkan ot & Roots & Kidney stones & Decoction & Int. \\
\hline $\begin{array}{l}\text { Rubus canescens DC. var. canescens } \\
\text { (Rosaceae), MARE } 9473\end{array}$ & Böğürtlen, Börtlen & $\begin{array}{l}\text { Fruits } \\
\text { Fruits }\end{array}$ & $\begin{array}{l}\text { Cancer } \\
\text { Intestinal diseases }\end{array}$ & $\begin{array}{l}\text { Infusion } \\
-\end{array}$ & $\begin{array}{l}\text { Int. } \\
\text { Eaten }\end{array}$ \\
\hline $\begin{array}{l}\text { Rubus sanctus Schreber } \\
\text { (Rosaceae), MARE } 9421\end{array}$ & $\begin{array}{l}\text { Böğürtlen, Börtlen } \\
\text { Karamık }\end{array}$ & $\begin{array}{l}\text { Fruits \& leaves } \\
\text { Fruits \& leaves }\end{array}$ & $\begin{array}{l}\text { Cancer } \\
\text { Intestinal diseases }\end{array}$ & $\begin{array}{l}\text { Infusion } \\
\text { Infusion }\end{array}$ & $\begin{array}{l}\text { Int. } \\
\text { Int. }\end{array}$ \\
\hline $\begin{array}{l}\text { Rumex crispus L. } \\
\text { (Polygonaceae), MARE } 9424\end{array}$ & Labada & Leaves & Digestive & Cooked & Int. \\
\hline $\begin{array}{l}\text { Sambucus ebulus L } \\
\text { (Caprifoliaceae), MARE 9422, } 10272\end{array}$ & Sultan otu & $\begin{array}{l}\text { Aerial parts } \\
\text { Seeds }\end{array}$ & $\begin{array}{l}\text { Chicken mite } \\
\text { Constipation }\end{array}$ & $\begin{array}{l}- \\
-\end{array}$ & $\begin{array}{l}\text { Put on the ground in coop } \\
\text { Int. }\end{array}$ \\
\hline
\end{tabular}

$\Delta$ Cultivated plant; + mixed with; Int. = internal use; Ext. = external use; $2 \times 1$ = twice a day; it means 1 glass of decoction or infusion indicated. 
TABLE 1. Folk medicinal plants of Lalapaşa (Edirne, Turkey) (continued)

\begin{tabular}{|c|c|c|c|c|c|}
\hline Botanical name and Voucher specimen & Local names & Parts used & Uses/Ailments treated & Preparations & Modes of use \\
\hline $\begin{array}{l}\text { Sambucus nigra L. } \\
\text { (Caprifoliaceae), MARE } 10275\end{array}$ & Mürver ağacı & $\begin{array}{l}\text { Flowers } \\
\text { Flowers } \\
\text { Flowers } \\
\text { Flowers }\end{array}$ & $\begin{array}{l}\text { Abdominal pain } \\
\text { Anthelmentic } \\
\text { Diabetes } \\
\text { Antitussive }\end{array}$ & $\begin{array}{l}\text { Decoction } \\
\text { Decoction } \\
\text { Decoction } \\
\text { Decoction }\end{array}$ & $\begin{array}{l}\text { Int. } \\
\text { Int. } \\
\text { Int. } \\
\text { Int. }\end{array}$ \\
\hline $\begin{array}{l}\text { A Satureja hortensis L. } \\
\text { (Lamiaceae), MARE 9441, } 10301\end{array}$ & $\begin{array}{l}\text { Çıbrika, } \\
\text { Çobrisa }\end{array}$ & Aerial parts & Stomachache & Infusion & Int. \\
\hline $\begin{array}{l}\text { Teucrium chamaedrys L. } \\
\text { subsp. chamaedrys } \\
\text { (Lamiaceae), MARE } 10276\end{array}$ & $\begin{array}{l}\text { Kısacıkmahmut, } \\
\text { Kösemahmut }\end{array}$ & Aerial parts & Cirrhosis & Infusion & Int. \\
\hline $\begin{array}{l}\text { Teucrium polium L. } \\
\text { (Lamiaceae), MARE } 9430\end{array}$ & Mayasıl otu & $\begin{array}{l}\text { Aerial parts } \\
\text { Aerial parts }\end{array}$ & $\begin{array}{l}\text { Hemorrhoids } \\
\text { Boil (on hand) }\end{array}$ & $\begin{array}{l}\text { Decoction } \\
\text { Decoction }\end{array}$ & $\begin{array}{l}\text { Int., after meal } \\
\text { Ext. }\end{array}$ \\
\hline $\begin{array}{l}\text { Thymus longicaulis C.Persl } \\
\text { subsp. Iongicaulis } \\
\text { var. subisophyllus (Borbas) Jalas } \\
\text { (Lamiaceae), MARE } 10263\end{array}$ & $\begin{array}{l}\text { Kekik otu, } \\
\text { Keklik otu, } \\
\text { Yeşil kekik otu }\end{array}$ & Aerial parts & Abdominal pain & Decoction & Int. \\
\hline $\begin{array}{l}\text { Typha latifolia L. } \\
\text { (Typhaceae), MARE } 10270\end{array}$ & $\begin{array}{l}\text { Kesik otu, } \\
\text { Saz otu }\end{array}$ & $\begin{array}{l}\text { Female } \\
\text { inflorescence }\end{array}$ & Haemostatic & - & Ext., wrapped in a cloth \\
\hline $\begin{array}{l}\text { Ulmus minor Miller subsp. canescens } \\
\text { (Melville) Browicz et Zieliński } \\
\text { (Ulmaceae), MARE } 9409\end{array}$ & Kara ağaç & Stem bark & Hand chaps & Decoction & Ext., $2 \times 1,60$ days \\
\hline \multirow[t]{5}{*}{$\begin{array}{l}\text { Urtica dioica L. } \\
\text { (Urticaceae), MARE 9412, } 9437\end{array}$} & Isırgan & Whole plants & Hypertansion & $\begin{array}{l}\text { Decoction, } \\
\text { before } \\
\text { breakfast }\end{array}$ & Int. \\
\hline & & Aerial parts & Stomachache & Cooked & Int. \\
\hline & & Aerial parts & Wound (on hand) & Infusion & Int. \\
\hline & & Aerial parts & Stomachache & Infusion & Int. \\
\hline & & Aerial parts & Allergy & Infusion & Int. \\
\hline $\begin{array}{l}\text { Urtica urens L. } \\
\text { (Urticaceae), MARE } 10268\end{array}$ & Isırgan & Aerial parts & Eczema & Decoction & Int. \\
\hline $\begin{array}{l}\text { Verbascum macrurum Ten. } \\
\text { (Scrophulariaceae), MARE } 9461\end{array}$ & Sığırkuyruğu & Flowers & Stomachache & Infusion & Int. \\
\hline $\begin{array}{l}\text { Verbascum ovalifolium Donn ex Sims } \\
\text { subsp. thracicum (Velen.) Murb. } \\
\text { (Scrophulariaceae), MARE } 10250\end{array}$ & Sığırkuyruğu & Flowers & Stomach ulcer & Infusion & Int. \\
\hline
\end{tabular}

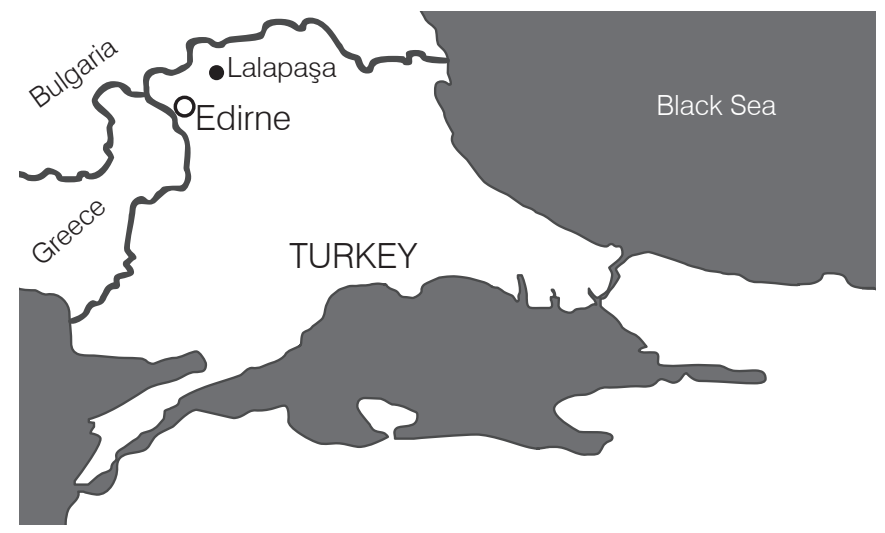

FIGURE 1. Location of Lalapaşa 
TABLE 2. The comparison of the folk medicinal plants recorded in the ethnobotanical studies made in the European part of Turkey ( $\neq$ : different usage, 一: plants not recorded)

\begin{tabular}{|c|c|c|c|c|c|}
\hline Botanical name & Lalapaşa (Edirne) & $\begin{array}{l}\text { Babaeski } \\
\text { (Kırklareli) }\end{array}$ & $\begin{array}{l}\text { Kırklareli } \\
\text { (except Babaeski) }\end{array}$ & Tekirdağ & Çatalca (İstanbul) \\
\hline $\begin{array}{l}\text { Achillea crithmifolia } \\
\text { Waldst. et Kit. }\end{array}$ & Hemorrhoids & Hemorrhoids & - & - & - \\
\hline Allium sativum $\mathrm{L}$. & $\neq$ & - & $\neq$ & - & - \\
\hline $\begin{array}{l}\text { Centaurea solstitialis L. } \\
\text { subsp. solstitialis }\end{array}$ & Malaria & Malaria & - & - & - \\
\hline Cotinus coggyria Scop & Eczema & $\begin{array}{l}\text { Eczema } \\
\text { Hemorrhoids }\end{array}$ & $\begin{array}{l}\text { Eczema } \\
\text { Hemorrhoids }\end{array}$ & $\neq$ & Hemorrhoids \\
\hline Cornus mas $\mathrm{L}$. & $\neq$ & $\neq$ & $\neq$ & - & $\neq$ \\
\hline $\begin{array}{l}\text { Corylus avellana } \mathrm{L} . \\
\text { var. avellana }\end{array}$ & Prostate ailments & - & Prostate ailments & - & - \\
\hline $\begin{array}{l}\text { Crataegus monogyna Jacq. } \\
\text { subsp. monogyna }\end{array}$ & $\neq$ & - & $\neq$ & - & $\neq$ \\
\hline Cydonia oblonga Miller & Cold & $\begin{array}{l}\text { Antitussive } \\
\text { Cold }\end{array}$ & $\neq$ & - & Cold \\
\hline $\begin{array}{l}\text { Ecballium elaterium (L.) A. } \\
\text { Rich. }\end{array}$ & $\begin{array}{l}\text { Sinusitis } \\
\text { Rheumatism } \\
\text { Hemorrhoids }\end{array}$ & $\begin{array}{l}\text { Sinusitis } \\
\text { Rheumatism } \\
\text { Hemorrhoids }\end{array}$ & $\begin{array}{l}\text { Sinusitis } \\
\text { Rheumatism } \\
\text { Hemorrhoids }\end{array}$ & $\begin{array}{l}\text { Rheumatism } \\
\text { Hemorrhoids }\end{array}$ & $\begin{array}{l}\text { Sinusitis } \\
\text { Hemorrhoids }\end{array}$ \\
\hline $\begin{array}{l}\text { Euphorbia nicaeensis All. } \\
\text { subsp. glareosa (Pallas ex Bieb.) A.R. Smith } \\
\text { var. lasiocarpa Boiss. }\end{array}$ & $\neq$ & - & $\neq$ & - & - \\
\hline Ficus carica L. subsp. carica & $\begin{array}{l}\text { Hemorrhoids } \\
\text { Wart }\end{array}$ & - & - & - & $\begin{array}{l}\text { Hemorrhoids } \\
\text { Wart }\end{array}$ \\
\hline $\begin{array}{l}\text { Fraxinus ornus } L \text {. } \\
\text { subsp. ornus }\end{array}$ & $\neq$ & - & $\neq$ & - & $\neq$ \\
\hline Hyoscyamus niger $\mathrm{L}$. & $\neq$ & - & $\neq$ & - & - \\
\hline Hypericum perforatum L. & $\begin{array}{l}\text { Diabetes } \\
\text { Wound } \\
\text { Kidney ailments }\end{array}$ & Kidney ailments & $\begin{array}{l}\text { Diabetes } \\
\text { Wound } \\
\text { Kidney ailments }\end{array}$ & $\neq$ & - \\
\hline Juglans regia L. & Hemorrhoids & $\neq$ & Hemorrhoids & - & - \\
\hline $\begin{array}{l}\text { Matricaria chamomilla } \mathrm{L} \text {. } \\
\text { var. recutita (L.) Grierson }\end{array}$ & $\neq$ & $\neq$ & $\neq$ & - & - \\
\hline Malva sylvestris L. & $\neq$ & $\neq$ & $\neq$ & $\neq$ & $\neq$ \\
\hline Melissa officinalis L. & $\neq$ & $\neq$ & $\neq$ & - & - \\
\hline Morus alba L. & $\neq$ & $\neq$ & - & - & $\neq$ \\
\hline $\begin{array}{l}\text { Origanum vulgare } \mathrm{L} . \\
\text { subsp. vulgare }\end{array}$ & $\begin{array}{l}\text { Stomach ailments } \\
\text { Cold }\end{array}$ & - & $\begin{array}{l}\text { Stomach ailments } \\
\text { Cold }\end{array}$ & $\neq$ & $\neq$ \\
\hline Paliurus spina-christi Miller & Kidney stones & Kidney stones & $\neq$ & $\neq$ & $\neq$ \\
\hline Papaver rhoeas $\mathrm{L}$. & $\neq$ & $\neq$ & $\neq$ & - & $\neq$ \\
\hline Pinus sylvestris $\mathrm{L}$. & Asthma & Asthma & Asthma & - & - \\
\hline Plantago lanceolata $\mathrm{L}$. & Diarrhea & - & $\neq$ & - & Diarrhea \\
\hline $\begin{array}{l}\text { Plantago major L. } \\
\text { subsp. major }\end{array}$ & Diabetes & $\neq$ & Diabetes & - & $\neq$ \\
\hline $\begin{array}{l}\text { Prunus spinosa L. } \\
\text { subsp. dasyphylla (Schur) Domin }\end{array}$ & Diabetes & Diabetes & Diabetes & Diabetes & Diabetes \\
\hline Robinia pseudoacacia L. & Bronchitis & - & - & - & Bronchitis \\
\hline Rosa canina $\mathrm{L}$. & $\begin{array}{l}\text { Asthma } \\
\text { Cold }\end{array}$ & Cold & Cold & Asthma & Asthma \\
\hline $\begin{array}{l}\text { Rubus canescens DC. } \\
\text { var. canescens }\end{array}$ & $\neq$ & - & $\neq$ & - & - \\
\hline Rubus sanctus Schreber & Cancer & $\neq$ & - & $\neq$ & Cancer \\
\hline Rumex crispus L. & $\neq$ & - & $\neq$ & - & $\neq$ \\
\hline Sambucus ebulus L & $\neq$ & $\neq$ & $\neq$ & $\neq$ & $\neq$ \\
\hline Sambucus nigra L. & Diabetes & - & - & $\neq$ & Diabetes \\
\hline Satureja hortensis L. & $\neq$ & - & - & $\neq$ & - \\
\hline $\begin{array}{l}\text { Teucrium chamaedrys } \\
\text { L. subsp. chamaedrys }\end{array}$ & $\neq$ & - & $\neq$ & - & - \\
\hline Teucrium polium L. & $\neq$ & - & $\neq$ & $\neq$ & - \\
\hline $\begin{array}{l}\text { Thymus longicaulis } \\
\text { C.Persl subsp. Iongicaulis } \\
\text { var. subisophyllus (Borbas) Jalas }\end{array}$ & Abdominal pain & $\neq$ & Abdominal pain & - & - \\
\hline Urtica dioica L. & $\begin{array}{l}\text { Hypertansion } \\
\text { Stomachache }\end{array}$ & $\neq$ & $\begin{array}{l}\text { Hypertansion } \\
\text { Stomachache }\end{array}$ & - & Stomach ache \\
\hline Urtica urens L. & $\neq$ & - & - & $\neq$ & - \\
\hline
\end{tabular}




\section{Türkiye'nin halk ilacı bitkileri, VIII: Lalapaşa (Edirne)}

ÖZET: Bu çalışmada Lalapaşa (Edirne) yöresinin halk ilacı olarak kullanılan bitkileri araştırılmıştır. Bilgiler, arazi çaIışmaları sırasında yerel halk hekimlerinden, deneyimli yaşlı kimselerden ve hastalardan kişisel görüşmelerle derlenmiş ve yörede kullanılan bitkilerin örnekleri toplanmıştır. Bu örneklerin teşhislerinin sonucunda, Lalapaşa yöresinde 55 bitkinin tedavi amacıyla kullanıldığı saptanmıştır. Bunlar metin içinde tablo halinde sunulmuştur. Bunların arasında 44 takson yabani ve 11 takson ise yörede yetiştirilen bitkilerdir. Halk ilacı olarak kullanılan bu bitkiler en çok mide rahatsızlıklarının giderilmesinde, hemoroidlere karşı, şeker hastalığının tedavisinde, soğuk algınlığının giderilmesinde ve siğillere karşı kullanılmaktadır.

ANAHTAR KELIMELER: Halk ilacı, Tıbbi bitkiler, Lalapaşa, Edirne, Türkiye

\section{REFERENCES}

1. Lalapaşa İlçesi Turizm Envanter Bilgileri, Lalapaşa Kaymakamlığı, 2003.

2. Davis PH. Flora of Turkey and the East Aegean Islands. vol. 1-9, Edinburgh University Press, Edinburgh, 19651985.

3. Tuzlacı E, Alparslan DF. Turkish folk medicinal plants partV:Babaeski (Kırklareli). J Fac Pharm Istanbul, 39:1123, 2007.
4. Kültür Ş. Medicinal plants used in Kırklareli Province (Turkey). J Ethnopharmacol, 11:341-364, 2007.

5. Akalın E, Alpınar K. Tekirdağ'ın tıbbi ve yenen yabani bitkileri hakkında bir araştırma. Ege Üniversitesi Eczacılik Fak Derg, 2:1-11, 1994.

6. Ecevit Genç G, Özhatay N. An ethnobotanical study in Çatalca (European part of Istanbul) II. Turkish J Pharm Sci, 3: 73-89, 2006. 